\title{
Étude ultrastructurale du cysticercoïde de Hymenolepis stylosa (Cestoda, Cyclophyllidea)
}

\author{
par CI. GABRION * et J.-M. VERDIER ** \\ * Laboratoire de Parasitologie comparée, Université des Sciences et Techniques \\ du Languedoc, F 34060 Montpellier Cedex. \\ ** Laboratoire d'Ecologie médicale et pathologie parasitaire, \\ Faculté de Médecine, rue Auguste-Broussonnet, F 34000 Montpellier.
}

\section{Résumé.}

L'observation au microscope électronique des structures tégumentaires du cysticercoïde d'H. stylosa et leur comparaison avec celles d'H. diminuta montrent une grande diversité entre ces deux larves.

Chez $H$. stylosa, le tégument du scolex et de la paroi cystique externe est couvert de microtriches de type classique; au niveau du pore antérieur, l'ornementation change brutalement, les microtriches sont flexueuses et sur la paroi cystique externe, seules sont visibles des expansions dans lesquelles se rassemble un matériel dense. Le cercomère enfin est couvert de microvillosités. La structure tégumentaire du cysticercoïde d' $H$. diminuta diffère de celle d'H. stylosa par l'absence de crochets au niveau du rostre et la présence de microvillosités sur la paroi cystique externe.

Ces observations constituent un argument supplémentaire en faveur de l'existence chez les larves cysticercoödes d'un gradient de différenciation plus ou moins marqué selon les espèces et qui affecterait tout ou partie du tégument, à l'exception du cercomère. Ce gradient résulterait d'inductions successives n'affectant chacune qu'une partie du cysticercoïde; il détermine (au moins en partie) la morphologie du scolex de l'adulte.

\section{Summary.}

On the ultrastructure of the cysticercoid of Hymenolepis stylosa (Cestoda : Cyclophyllidea).

Electron microscopy of the tegument of $H$. stylosa cysticercoid as compared with that of $H$. diminuta reveals great variations in the fine structure of the larval tegument of these two species.

Reçu le 4 octobre 1977. 
On the scolex and the inner cyst wall, the tegument of $H$. stylosa cysticercoid bears typical microtriches; at the level of the anterior pore, the microtriches are flexuous. The tegument of the outer cyst wall appears as a syncytial band with dense material which accumulates in the region below the unit membrane, and the tegument of the cercomer is increased by branchied microvillies. While, the scolex of $H$. diminuta cysticercoid is unarmed and the tegument of the outer cyst wall bears branched microvillies.

The data permit the conclusion that the differenciation of tegumental structures is graduated from the scolex to the cercomer.

This differenciation is more or less important according to the parasite and results of successive inductions which affect parts of the cysticercoid.

The significance of these findings is discussed in terms of the possible function of these structures and the penetration of substances.

Les travaux réalisés en microscopie électronique sur Hymenolepis diminuta Rudolphi, 1819 par Ubelaker, Cooper et Allison, 1970 ; Allison, Ubelaker et Cooper, 1972 ; Cooper, Ubelaker et Allison, 1975 montrent des différences importantes entre la structure du cysticercoïde de ce Cestode et celle des autres Cyclophyllides étudiés jusqu'à ce jour (Baron, 1971 ; Rees, 1973 ; Caley, 1974 ; Caley, 1976 ; Crowe, Burt et Scott, 1974; Gabrion et Gabrion, 1976). En outre, chez H. diminuta, le caractère «externe» (avant invagination) ou 《interne» (après invagination) de la différenciation du scolex a été controversé (Voge et Heyneman, 1957; Schiller, 1959). Enfin, $H$. diminuta, bien que placé dans le genre Hymenolepis, présente la particularité d'avoir un rostre inerme.

Nous avons pensé qu'il serait intéressant d'étudier un Hymenolepididae dont l'anatomie correspond exactement à la diagnose de la famille et dont le développement larvaire est conforme à celui des autres cysticercoïdes afin de confronter ces observations avec celles réalisées chez $H$. diminuta.

Pour cela, nous avons étudié les ultrastructures du cysticercoïde infestant d'H. stylosa (Rudolphi, 1809) Raillet, 1899, parasite de Corvidés. Cet Hymenolepis possède tous les caractères des Hymenolepididae, en partıculier, un scolex dont le rostre est armé d'une couronne de 10 crochets. D'autre part, nous avons montré lors de la formation du cysticercoïde de ce Cestode (Gabrion, 1977) que le scolex se différencie avant invagination dans la vésicule cystique.

\section{Matériel et Méthodes}

Les adultes d'H. stylosa proviennent de Choucas (Coloeus monedula) capturés en Camargue. Ceux d'H. diminuta ont été prélevés chez des Mulots (Apodemus sylvaticus) piégés dans les Cévennes.

Dans les deux cas, les segments gravides ont servi à l'infestation expérimentale de lots de Tenebrio molitor (Coléoptère, Tenebrionide). 
Pendant la durée de l'expérimentation, les Insectes sont maintenus dans une enceinte climatique à $28^{\circ} \mathrm{C}$.

Après dissection des Tenebrio, les cysticercoïdes retirés de l'hémocoele sont fixés pendant 16 heures par immersion dans du glutaraldéhyde à $4 \%$ tamponné au cacodylate à $0,1 \mathrm{M}$, pH 7,4.

\section{Résultats}

A. Etude ultrastructurale du cysticercoïde mûr d'H. stylosa.

Le cysticercoïde infestant d'H. stylosa (fig. l) est ovoïde et mesure en moyenne $330 \mu \mathrm{m}$ de longueur et $220 \mu \mathrm{m}$ de largeur. Le métacestode, mobile, est situé à l'intérieur de la cavité d'invagination limitée par la paroi interne du cysticercoïde. La vésicule cystique plus ou moins oblitérée sépare cette paroi de la paroi externe.

Le scolex du métacestode comprend quatre ventouses et un rostre armé de 10 crochets identiques, de $32 \mu \mathrm{m}$ de longueur. Un cou relie le scolex à la paroi cystique interne.

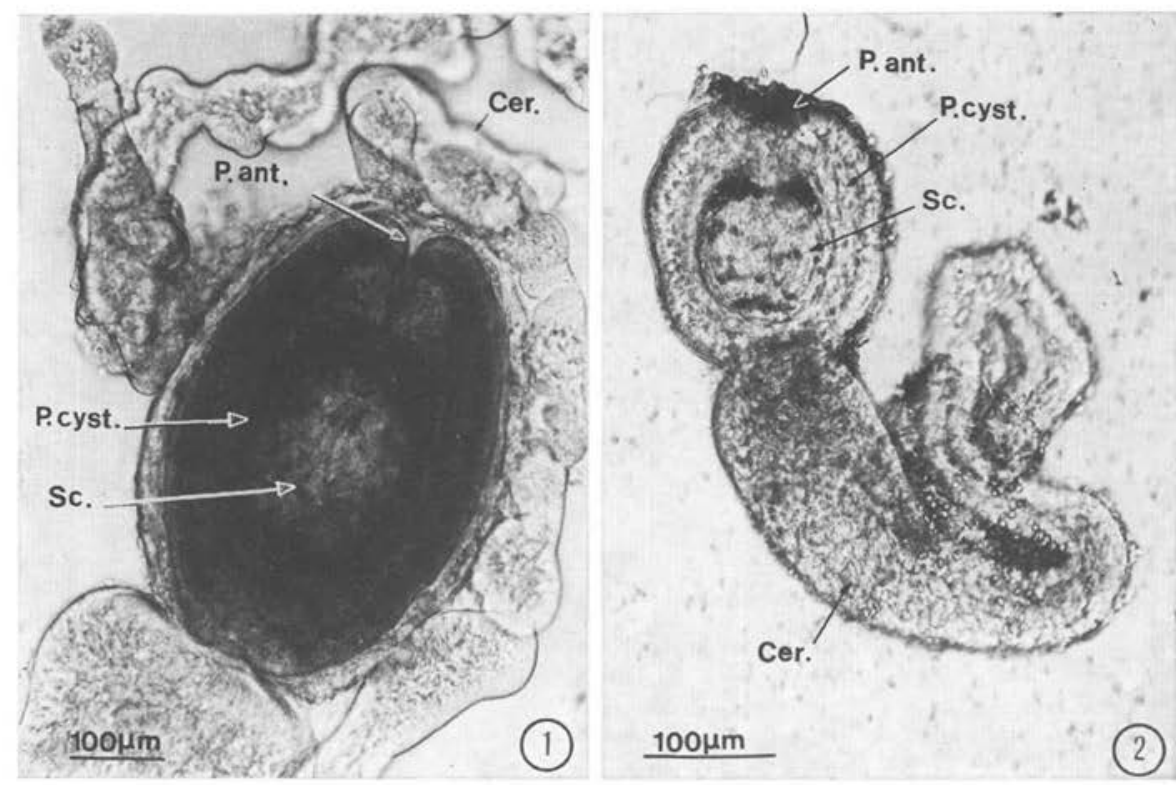

FIG. 1. - Cysticercoïde d'Hymenolepis stylosa.

FIG. 2. - Cysticercoïde d'Hymenolepis diminuta. Cer.: Cercomère ; P. ant. : Pore antérieur ; P. cyst.: Parois cystiques; Sc. : Scolex. 
Le pore antérieur reste ouvert et met en communication la cavité d'invagination avec l'hemocoele de l'Insecte.

Au cysticercoïde est appendu un cercomère long et flexueux qui peut atteindre $2,7 \mathrm{~mm}$.

La larve d'H. stylosa, libre dans l'hemocoele, est le plus souvent localisée dans le corps adipeux qui entoure le mésentéron. Le cercomère est alors étroitement accolé au tube digestif.

\section{1. - LE SCOLEX.}

Le syncytium tégumentaire du scolex est recouvert sur toute sa surface de micro. triches semblables à celles décrites chez les Cestodes adultes (Jha et Smyth, 1969). Elles se composent d'une partie distale, pointue, dense aux électrons, inclinée dans le sens antéro-postérieur, et d'une partie proximale qui est une invagination du cytoplasme tégumentaire. Les deux parties sont séparées par une plaque basale multilaminée. On n'observe pas de glycocalyx à la surface de ces microtriches. Le cytoplasme du syncytium qui couvre le scolex, y compris les ventouses et le rostre, est occupé par de nombreuses vésicules claires, contenant un matériel dense aux électrons (fig. 3, 4 et 5). Il est relié aux cellules sous-tégumentaires par des canaux de connexion au niveau desquels s'infléchit la membrane basale. Chaque ventouse (fig. 3), complètement entourée par une membrane basale, constitue une unité organique à l'intérieur du scolex. La musculature en est très complexe. On distingue de l'extérieur vers l'intérieur :

- La membrane basale externe, contiguë à la lame basale du syncytium tégumentaire qui recouvre le scolex,

- Des fibres musculaires circulaires et longitudinales (par analogie avec la disposition des couches musculaires sous-tégumentaires),

- Des fibres musculaires radiaires, perpendiculaires aux précédentes et qui vont de la basale interne à la basale externe. Nous n'avons pas observé d'hémidesmosomes reliant les fibres musculaires radiaires à la basale de la ventouse, comme cela a été observé par Featherston (1971) chez Taenia hydatigena,

- Enfin, contre la basale interne, un ensemble de fibres circulaires et longitudinales profondes.

Fig. 3. - H. stylosa: Structure de la ventouse. On peut noter la complexité de la musculature. B1. : Basale ; Cel. par.: Cellule parenchymateuse; F.m.c.: Fibres musculaires circulaires; F.m.l.: Fibres musculaires longitudinales; F.m.r.: Fibres musculaires radiaires; Mt.: Microtriches ; My.: Myoblaste ; S.t.: Syncytium tégumentaire ; Vés. : Vésicule claire.

Fig. 4. - H. stylosa: Structure du rostre (R.) et du sac rostral (S.r.); Cr. : Crochet.

FIG. 5. - H. stylosa: Coupe longitudinale du cysticercoïde mettant en évidence la succession des différentes parties constitutives de la larve. C.c. : Corpuscule calcaire ; Fbl. : Fibroblaste; Gx. : Glycocalyx ; P. cyst. ext. : Paroi cystique externe ; P. cyst. int. : Paroi cystique interne; Sc. : Scolex; Vés. cyst.: Vésicule cystique. 

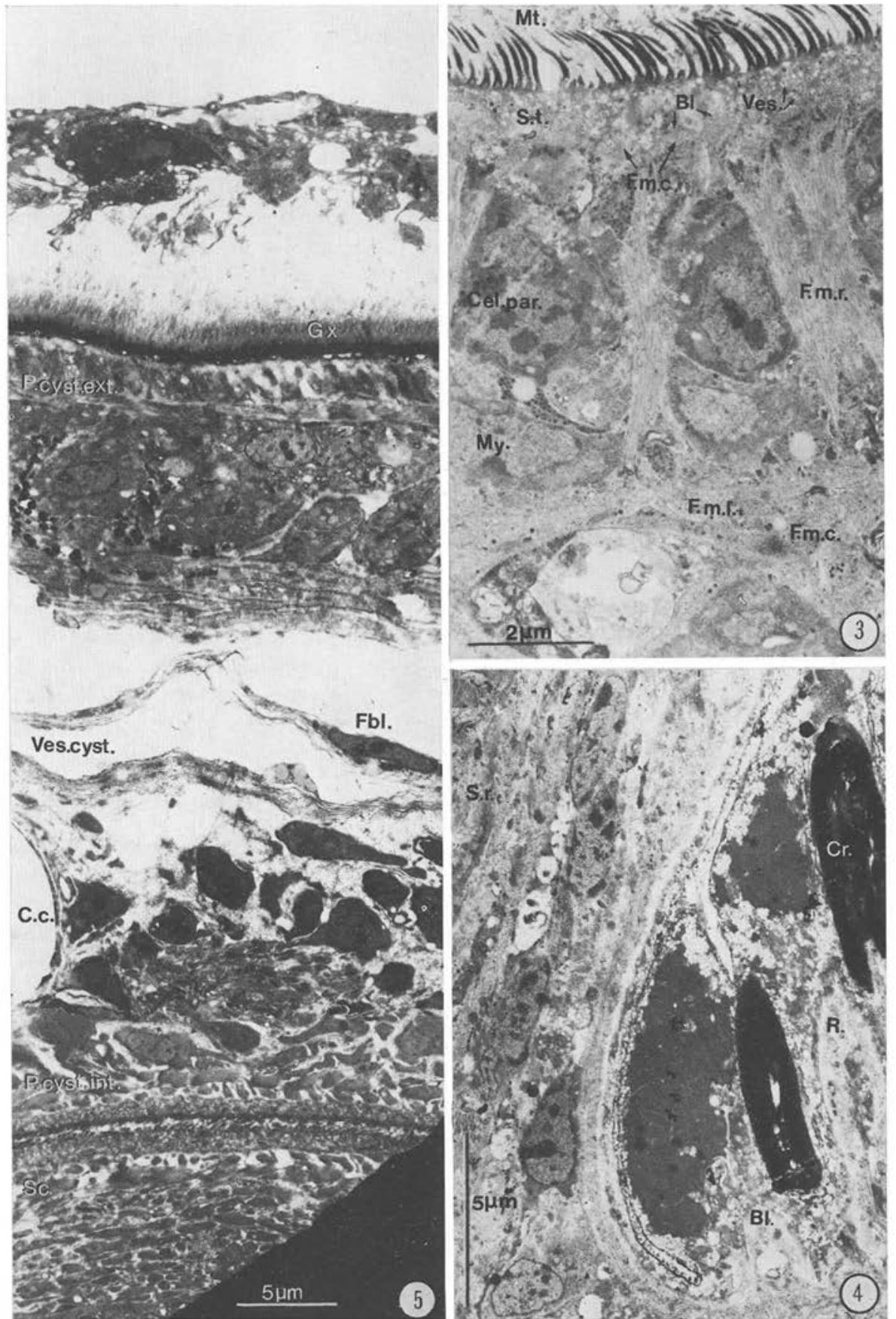
L'espace entre les fibres radiaires est occupé par différents corps cellulaires: cellules parenchymateuses à glycogène et myocytes. Ceux-ci, allongés, bordent la lame basale interne.

Au niveau des ventouses, le syncytium tégumentaire est bordé de microtriches dont la partie distale est longue et flexueuse, la partie proximale courte. Nous n'avons pas observé, à cet endroit, de cellules sous-tégumentaires, en relation avec le syncytium.

A l'intérieur du sac rostral, le rostre occupe une position centrale à l'extrémité du scolex, entre les quatre ventouses (fig. 4). Le syncytium tégumentaire qui borde le sac rostral et le rostre est pourvu de microtriches réduites et éparses.

Le rostre porte dix crochets dont la formation et la structure correspondent aux observations réalisées par Mount (1970) chez Taenia crassiceps. Chez H. stylosa cependant, il n'y a pas, comme le signalent Crowe, Burt et Scott (1974) chez Paricterotaenia paradoxa, d'accumulation de «rod like structures» ou «disc. "shape bodies» autour du crochet, mais une condensation importante de matériel opaque aux électrons.

L'ultrastructure du tégument du cou est très proche de celle du scolex, mais les microtriches, moins nombreuses, possèdent une partie proximale plus développée.

\section{2. - PARoi interne dU cysticercoïde.}

Le syncytium tégumentaire de la paroi cystique interne diffère peu de celui du métacestode. Il est toujours occupé par de nombreuses vésicules claires à corps dense. A ce niveau cependant, et à mesure que l'on se rapproche du pore antérieur, les microtriches deviennent moins nombreuses et leur structure se modifie (fig. 11 et 12). La partie distale n'est plus constituée par une courte «épine» dense aux électrons, mais par un filament dont l'extrémité se fragmente en donnant des vésicules, selon un processus identique à celui décrit par Lascano et al. (1975) chez Echinococcus granulosus. Ces vésicules s'accumulent dans la cavité d'invagination du cysticercoïde.

Sous la basale du syncytium (fig. 5), on observe les couches musculaires, circulaires et longitudinales, plus discrètes que dans le scolex et au-dessous, les cellules

Fig. 6. - H. stylosa: Structure de la paroi cystique externe au niveau du pore antérieur. On observe à l'intérieur des cellules sous-tégumentaires (Cel. tg.) la présence de nombreux granules (Gr.) et, dans le syncytium tégumentaire, le dépôt d'un matériel dense aux électrons. Gx.: Glycocalyx.

Fig. 7. - H. stylosa: Détail de la paroi cystique externe dans une région moyenne entre le pore antérieur et la base du cercomère. F.c.: Fibres collagènes; Gr.: Granules; My. : Myoblastes.

FIG. 8. - H. stylosa: Dispa:ition des microvillosités à la limite cercomère-cysticercoïde. C. con.: Canal de connexion; Mv.: Microvillosités.

Fig. 9. - H. stylosa: Dépôt du matériel dense à la périphérie du syncytium tégumentaire (S.t.) bordant la paroi cystique externe. F.c.: Fibres collagènes ; Gx.: Glycocalyx.

Fig. 10-11-12. - H. stylosa: Evolution de l'ornementation tégumentaire du pore antérieur (fig. 10) à la paroi cystique interne (fig. 11-12); Mt. fil.: Microtriche filamenteuse; Mt.: Microtriche. 

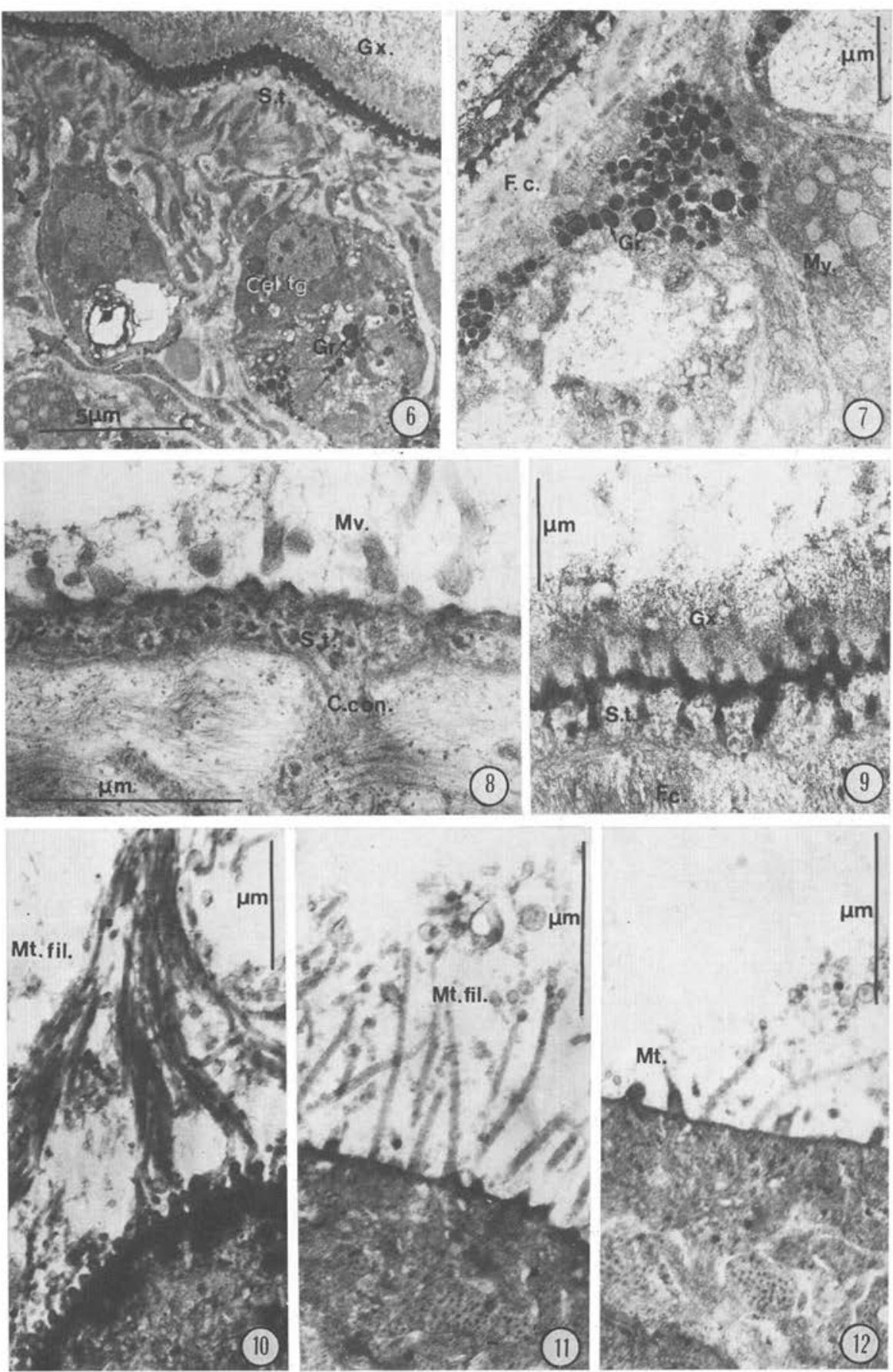
sous-tégumentaires et les myocytes. Les cellules parenchymateuses riches en glycogène, les cellules à corpuscules calcaires et des éléments du système excréteur sont localisés contre la cavité de la vésicule cystique; celle-ci est délimitée par d'étroits fibroblastes (cellules bipolaires) dont les prolongements traversent la cavité et viennent s'appliquer sur la paroi cystique externe. Dans le noyau allongé de ces cellules bipolaires, la chromatine est condensée en plaques contre l'enveloppe nucléaire. Leur cytoplasme est pourvu de nombreux ribosomes. Le R.E.L. et le R.E.r. sont très développés et les mitochondries abondantes. Par endroits, on peut observer des gouttelettes lipidiques.

\section{3. - Le PORE ANTÉRIEUR.}

Au niveau du pore antérieur, l'allure du syncytium tégumentaire change brutalement (fig. 10). La membrane basale n'est plus visible et le cytoplasme est envahi par une substance dense aux électrons. La surface est couverte de microtriches longues et flexueuses très serrées qui diffèrent essentiellement des microvillosités par le fait qu'elles ne sont jamais ramifiées et que le filament dense est bien distinct de la partie proximale. A l'intérieur de la partie flexueuse on observe des microfilaments longitudinaux. Les microtriches sont groupées en faisceaux par les éléments du glycocalyx. $\mathrm{Au}$ fur et à mesure que l'on s'éloigne du pore, les filaments disparaissent, il ne reste sur le tégument que des expansions denses dont on peut difficilement analyser la structure.

\section{4. - Paroi externe du cysticercoïde.}

La paroi externe de même épaisseur que la paroi interne est caractérisée par une succession de quatre zones (fig. 5).

La première, la plus interne, est constituée par des fibroblastes. C'est à ce niveau que s'observent les cellules à corpuscules calcaires. Au-dessus, les prolongements cytoplasmiques des fibres sont plus larges et enserrent par endroits les cellules du parenchyme.

FIG. 13. - H. stylosa: Structure du cercomère. A l'intérieur des canaux de connexion, on observe des mitochondries (M.) en très grand nombre, orientées perpendiculairement à la surface du tégument.

Fig. 14. - H. stylosa: Détail de la surface du tégument montrant la structure des microvillosités (Mv.); S.t.: Syncytium tégumentaire.

Fig. 15. - H. stylosa: Présence dans le cercomère de deux types cellulaires: d'une part les cellules sous-tégumentaires (Cel. tg.); d'autre part les myoblastes (My.) que l'on voit ici en relation avec des fibres musculaires longitudinales.

Fig. 16. - H. stylosa : «cellule astéroïde » ou « $\beta$ cell » selon les auteurs, dans le cercomère.

Fig. 17-18-19. $-H$. diminuta: Différents types d'ornementation tégumentaire.

Fig. 17: Microtriches au niveau du scolex (Sc.) et de la paroi cystique interne (P. cyst. int.).

Fig. 18: Microtriches filamenteuses (Mt. fil.) au niveau du pore antérieur (P. ant.).

Fig. 19: Microvillosités ramifiées (Mv.) dans le cercomère. S.t.: Syncytium tégumentaire; Vés. : Vésicule claire. 


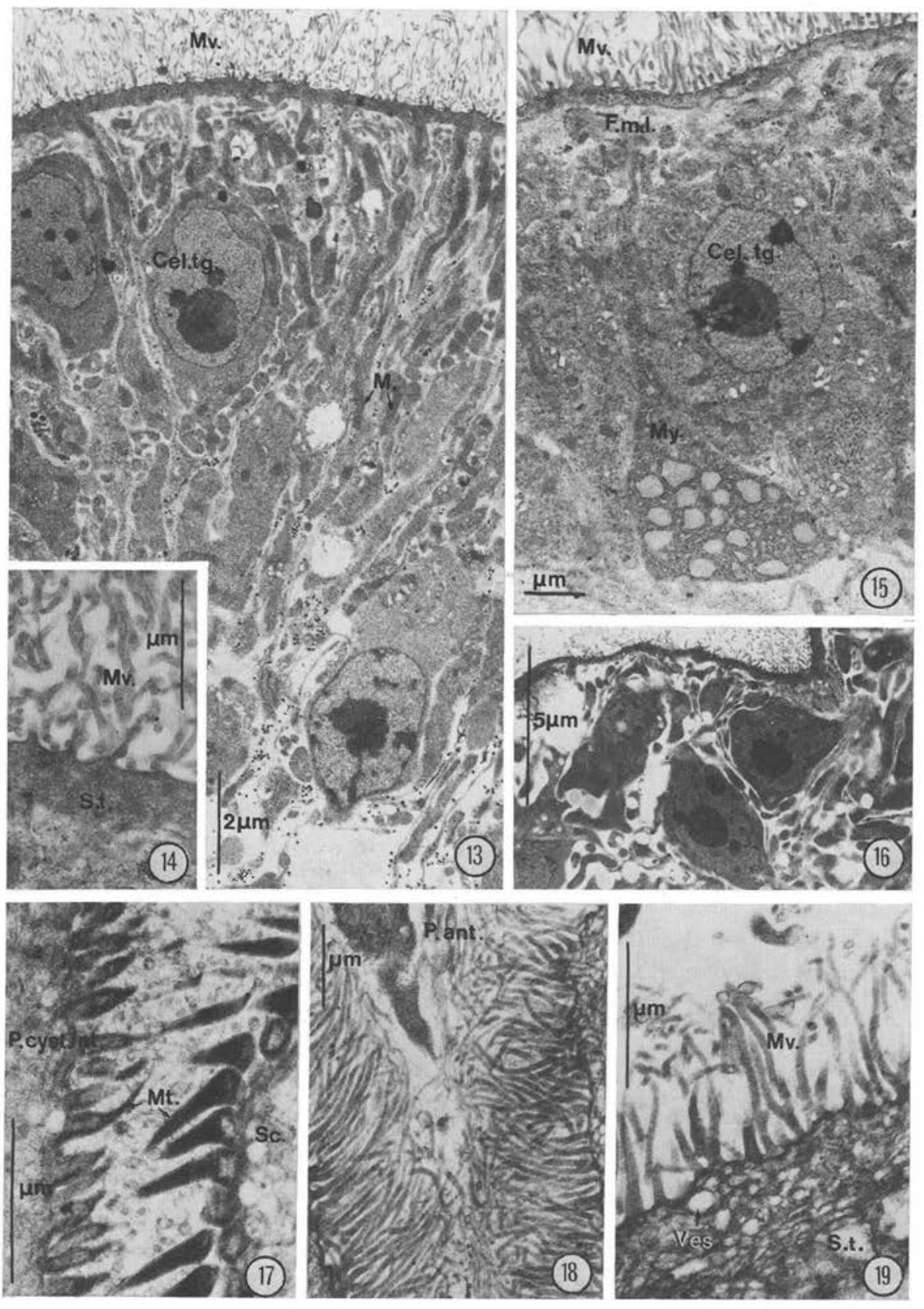


La couche suivante présente deux types cellulaires. L'un est constitué far les cellules sous-tégumentaires, reliées au syncytium superficiel par les canaux de connexion, l'autre par les cellules sécrétrices.

Dans les cellules sous-tégumentaires, les noyaux sont souvent rejetés à la périphérie. Le cytoplasme est occupé par de nombreux granules sphériques opaques aux électrons et des vésicules claires à l'intérieur desquelles s'observe un corps dense.

Les cellules sécrétrices correspondent aux myocytes décrits par Lumsden et Byram III (1967) chez Lacystorhynchus tenuis et aux cellules sécrétrices décrites par Ubelaker et al. (1970) chez H. diminuta.

Ces cellules sont caractérisées par la présence d'un noyau de forme irrégulière, peu dense aux électrons, dans lequel la chromatine est condensée à la périphérie ou dispersée dans le cytoplasme. La plupart du temps, il n'y a qu'un seul nucléole, excentré. L'enveloppe nucléaire présente de nombreux pores et la membrane externe, recouverte de ribosomes, est en continuité avec les citernes du R.E.r. Les complexes golgiens sont très abondants. Les mitochondries sont habituellement localisées à la périphérie du cytoplasme dans lequel le R.E.r. est l'organite prépondérant.

Dans la $3^{\circ}$ zone, se succèdent les couches musculaires tégumentaires longitudinales et circulaires. Les amas dispersés de fibres circulaires apparaissent au sein du tissu fibreux constitué de fibres étroites entrelacées, incluses dans une matrice homogène finement granuleuse.

Le syncytium superficiel ( $4^{e}$ zone) est limité vers l'intérieur par une membrane basale qui s'infléchit au niveau des canaux de connexion.

La structure du syncytium de la paroi cystique externe est particulière.

On peut suivre les modifications qui apparaissent depuis le pore antérieur jusqu'au cercomère (fig. $6,7,8$ et 9 ).

Au niveau du pore antérieur (fig. 6), une substance dense occupe le syncytium, les vésicules claires et les autres organites du cytoplasme ont disparu, la lame basale n'est pas visible; à la surface apparaissent des expansions sans structure particulière. A mesure que l'on se rapproche du cercomère, le dépôt de substance dense est moins prononcé. Les expansions du tégument s'estompent. On observe la migration de la substance dense de la partie profonde vers la périphérie où elle se dépose, au niveau de la membrane plasmique. Ce transport ne semble pas s'effectuer à partir de «rodlike structure», comme dans le cas de la croissance des microtriches.

Le glycocalyx qui recouvre le tégument est très abondant et constitue un filet protecteur entre la larve cysticercoïde et les hémocytes de l'Insecte.

FIG. 20-21. $-H$. diminuta: Montage d'une coupe longitudinale du cysticercoïde mettant en évidence la structure des différentes parties. Cel. tg. : Cellule sous-tégumentaire; Fbl. : Fibroblaste; F.c.: Fibre collagène ; F.m.c. : Fibres musculaires circulaires; F.m.l. : Fibres musculaires longitudinales; Mv.: Microvillosités; My.: Myoblastes; P. cyst. ext.: Paroi cystique externe; Sc. : Scolex ; S.t.: Syncytium tégumentaire; Vés. cyst.: Vésicule cystique. FIG. 22. - $H$. diminuta: Structure du cercomère. On observe les deux types cellulaires, cellule sous-tégumentaire (Cel. tg.) et myoblaste (My.) ; Mv. : Microvillosités. 

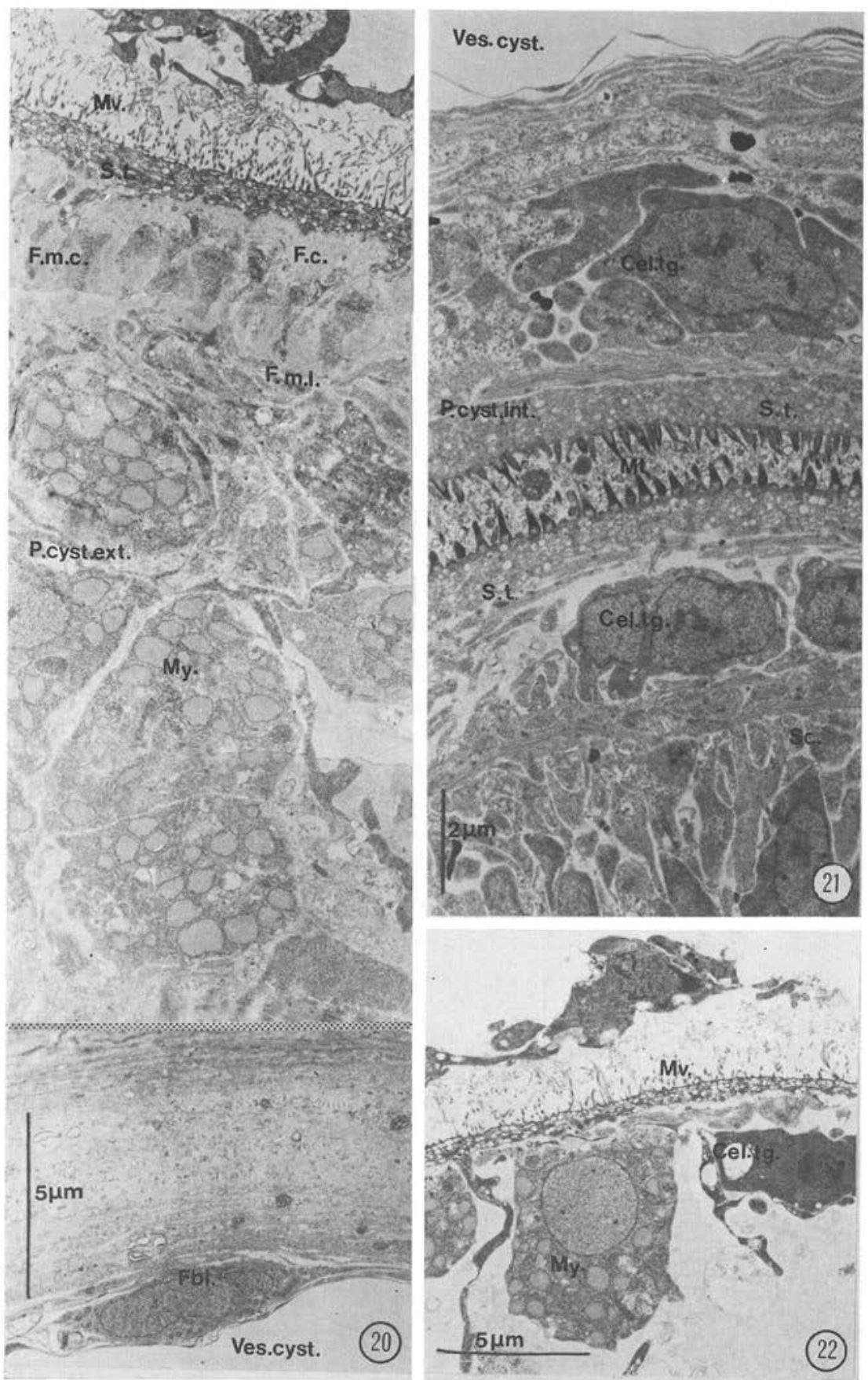


\section{5. - Le Cercomère (fig. 13, 14, 15 et 16).}

Nous n'avons pu déterminer de manière précise si l'accroissement du cercomèrc (comme celui du cysticercoïde) est dû à une caryocinèse des cellules tégumentaires initiales et à la différenciation du cytoplasme périnucléaire ou à l'apport d'une seconde population de cellules issues de cellules indifférenciées, de type « astéroïde » (Sakamoto et Sugimura, 1970) ou «Beta cell » des Planaires (Hay et Coward, 1975). Cependant, la présence de cellules indifférenciées à cytoplasme sombre au voisinage du tégument (fig. 16) nous incite à penser que l'allongement serait dû à un apport de ces cellules.

Le tégument du cercomère est recouvert de microvillosités, longues et parfois ramifiées à la base (fig. 14), semblables à celles qui recouvrent le syncytium tégumentaire du précysticercoïde d'H. citelli (Collin, 1970) ou d'A. constricta (Gabrion et Gabrion, 1975). Les microvillosités constituent un feutrage enserrant les cellules de l'hôte. Le syncytium superficiel du cercomère, étroit, est dépourvu de vésicules claires à corps dense. Les mitochondries sont disposées parallèlement à la basale. On observe la migration à travers les canaux de connexion des «rod-like-structures» provenant des cellules sous-tégumentaires.

Les cellules sous-tégumentaires possèdent un noyau lobé, pourvu d'un nucléole large, dense aux électrons. Le péricaryon étroit est pauvre en organites, à l'exception des mitochondries qui sont nombreuses et volumineuses (fig. 15).

Sous la basale, les fibres musculaires dispersées sont longitudinales. Les myocytes reliés aux fibres sont plus profonds et caractérisés par un R.E.r. abondant (fig. 15).

$\mathrm{Au}$ cours de l'allongement du cercomère, les espaces intercellulaires s'agrandissent et les mitochondries des cellules sous-tégumentaires augmentent en nombre et en taille.

\section{B. Etude de tégument du cysticercoïde mûr d'H. diminuta (fig. 17 à 22).}

Nos observations sur l'ultrastructure du cysticercoïde d'H. diminuta (fig. 20, 21, 22) correspondent à celles réalisées par Ubelaker, Cooper et Allison, 1970; Allison, Ubelaker et Cooper, 1972; Cooper, Ubelaker et Allison, 1975 et ne diffèrent pas fondamentalement de celles réalisées chez $H$. stylosa. Aussi, nous nous sommes limités aux structures du tégument où nous avons noté des différences importantes.

Le tégument du scolex est recouvert sur toute sa surface de microtriches semblables à celles observées chez $H$. stylosa (fig. 17). On n'observe pas de glycocalyx et le syncytium tégumentaire est occupé par de nombreuses vésicules claires de forme et de taille variables.

La paroi interne du cysticercoïde qui borde la vésicule cystique est réduite. Elle ressemble dans son organisation à celle du scolex. Cependant, comme chez H. stylosa, les microtriches du syncytium tégumentaire sont moins nombreuses et possèdent une partie proximale plus allongée et une partie distale plus claire (fig. 17).

Par contre, le revêtement de la paroi externe du cysticercoïde (du pore antérieur au cercomère) est très différent. Le syncytium tégumentaire porte des microvillosités longues et ramifiées et le cytoplasme, comme au niveau du scolex, est occupé par de 
nombreuses vésicules claires. Là encore, le glycocalyx n'est pas abondant. Ce revêtement est identique à celui du syncytium tégumentaire qui recouvre le cercomère (fig. 19).

$\mathrm{Au}$ niveau du pore antérieur, les villosités sont plus longues et plus serrées que sur la paroi cystique externe, mais elles ne présentent pas la structure particulière observée chez H. stylosa (fig. 18).

\section{Discussion}

L'étude ultrastructurale du cysticercoïde infestant d'H. stylosa met en évidence une grande diversité dans la structure du syncytium tégumentaire selon les différentes régions de la larve.

Au niveau du scolex, les microtriches sont semblables à celles décrites chez divers Cyclophyllides adultes (fig. 5); sur le rostre, certaines sont à l'origine des crochets (fig. 4).

Sur la paroi cystique interne qui borde la cavité d'invagination, jusqu'au pore antérieur, la structure des microtriches est très proche de celle qui couvre le tégument du scolex.

$\mathrm{Au}$ niveau du pore antérieur, l'ornementation du tégument change brutalement; on observe la présence de microtriches munies d'un long filament. Au-delà, il n’y a plus de microtriches mais seulement des expansions superficielles sans structure précise (fig. 6 à 12).

Enfin, au niveau du cercomère, le syncytium tégumentaire reste couvert de microvillosités fines et ramifiées (fig. 13 à 15 ).

La répartition de ces structures confirme l'existence d'un gradient de différenciation du syncytium tégumentaire que nous avions mis en évidence chez $A$. constricta (Euzet, Gabrion, 1976). Le scolex est la région qui subit la différenciation maximale ; à l'opposé, le tégument du cercomère est la partie de la larve qui semble la moins affectée par ce processus.

Cette diversité dans la structure du tégument du cysticercoïde infestant d'H. stylosa correspondrait à une série d'inductions qui, se succédant au cours du développement du cysticercoïde, n'affecteraient chaque fois qu'une partie de la larve.

Chez $H$. diminuta, l'étude de la larve infestante montre bien, au niveau du métacestode et de la paroi cystique interne la présence de microtriches (fig. 20, 21). Par contre, au niveau de la paroi cystique externe, le syncytium tégumentaire reste bordé, comme le cercomère, de microvillosités, la seule différence avec le tégument du précysticercoïde réside dans la plus grande épaisseur du syncytium (fig. 20, 22).

L'absence de crochets rostraux et la persistance de microvillosités sur la paroi cystique externe d'H. diminuta pourraient être en relation avec la durée du développement des larves. En effet, chez $H$. diminuta, le stade infestant est atteint en 14 jours à $28^{\circ} \mathrm{C}$, en 8 jours à $30^{\circ} \mathrm{C}$, alors que le cysticercoïde d' $H$. stylosa ne devient infestant qu'après 24 jours à $28^{\circ} \mathrm{C}$. Chez $H$. diminuta, l'apparition des microtriches de 
type classique au niveau du scolex et de la paroi interne du cysticercoïde se ferait avant la disparition des microvillosités sur le reste de la larve.

Les variations de structure du tégument selon les régions du cysticercoïde pourraient être en relation avec l'activité métabolique de ces différentes parties, les phénomènes d'absorption pouvant être en relation étroite avec la présence de microtriches. En effet, chez un Trématode adulte, Apatemon gracilis, Erasmus (1969) observe que les structures tégumentaires varient en fonction du degré d'activité sécrétoire et d'absorption. Smyth (1967) montre que chez l'adulte d'Echinococcus granulosus, l'absorption n'a lieu que dans la région pourvue de microtriches. D'autre part, Moczon $(1973 b$ ) et Bogitsh (1967) chez $H$. diminuta ont mis en évidence l'absence de phosphatases alcalines au niveau de la paroi externe du cysticercoïde; seules sont présentes les phosphatases acides non spécifiques. Ces auteurs considèrent que cette zone ne constitue pas un lieu d'absorption des matières nutritives. Par contre, le pore antérieur, la paroi cystique interne et le strobile sont des zones à forte activité.

En ce qui concerne l'absence d'ornementation sur le tégument de la paroi cystique externe chez $H$. stylosa, il pourrait s'agir, soit d'une induction trop faible, soit d'une modification du tégument sous l'action de l'hôte; des figures identiques ont en effet été obtenues par Borgers et al. (1975) sur des cysticerques de Taenia taeniaeformis traités au Mébendazole.

\section{Conclusion}

L'observation au microscope électronique des structures tégumentaires du cysticercoïde d' $H$. stylosa et leur comparaison avec celles d' $H$. diminuta montrent une grande diversité entre ces deux larves. Elles constituent un argument supplémentaire en faveur de l'existence, chez les larves cysticercoïdes, d'un gradient de différenciation antéro-postérieur. Ce gradient, plus ou moins marqué selon les espèces affecte tout ou partie du tégument, à l'exception du cercomère. Ce gradient détermine (au moins en partie) la morphologie du scolex de l'adulte; il résulterait d'inductions successives n'affectant chacune qu'une partie du cysticercoïde.

Une étude cytochimique des différentes régions du cysticercoïde d'H. stylosa, en particulier du pore antérieur qui constitue, du point de vue morphologique, une zone de transition importante, devrait nous permettre de vérifier l'existence de corrélations entre la structure du tégument, en particulier la nature des microtriches et un état physiologique déterminé.

\section{Bibliographie}

Allison (V. F.), Ubelaker (J. E.) et Cooper (N. B.), 1972. - The fine structure of the cysticercoid of Hymenolepis diminuta. II. The inner wall of the capsule. Z. Parasitenk. 39, 137-147. 
BARON (P. J.), 1971. - On the histology, histochemistry and ultrastructure of the cysticercoid of Raillietina cesticillus (Molin, 1858) Fuhrmann, 1920 (Cestoda, Cyclophyllidea). Parasitology, 62, 233-245.

Bogrtsh (B. J.), 1967. - Histochemical localization of some enzymes in cysticercoids of two species of Hymenolepis. Exp. Parasitol., 21, 373-379.

Borgers (M.), De Nollin (S.), Verheyen (A.), Vanpariss (O.) et Thienpont (D.), 1975. Morphological changes in cysticerci of Taenia taeniaeformis after mebendazole treatment. J. Parasitol., 61, 830-843.

CALEY (J.), 1974. - The functional significance of scolex retraction and subsequent cyst formation in the cysticercoid larva of Hymenolepis microstoma. Parasitology, 68, 207-227.

CALEY (F.), 1976. - Ultrastructural studies of the cysticercoid of Moniezia expansa (Anoplocephalidae) with special reference to the development of the cyst. Z. Parasitenk., 48, 251-262.

Collin (W. K.), 1970. - Electron microscopy of postembryonic stages of the tapeworm, Hymenolepis citelli. J. Parasitol., 56, 1159-1170.

Cooper (N. B.), Allison (V.F.) et Ubelaker (J. E.), 1975. - The fine structure of the cysticercoid of Hymenolepis diminuta. III. The scolex. Z. Parasitenk., 46, 229-239.

Crowe (D. G.), Burt (M. D. B.) et ScotT (J. S.), 1974. - On the ultrastructure of the polycercus larva of Paricterotaenia paradoxa (Cestoda: Cyclophyllidae). Can. J. Zool., $52,1397-1405$.

Erasmus (D. A.), 1969 b. - Studies on the host-parasite interface of strigeoid trematodes. V. Regional differentiation of the adhesive organ of Apatemon gracilis minor Yamaguti, 1933. Parasitology, 59, 245-256.

Euzet (L.) et Gabrion (C.), 1976. - Mise en évidence d'un gradient de différenciation du tégument chez la larve de deux Cestodes Cyclophyllides. C.R. Acad. Sci. Paris, 283, sér. D, 367-370.

FeAtherston (D. W.), 1972. - Taenia hydatigena. IV. Ultrastructure. study of the tegument. Z. Parasitenk., 38, 214-232.

GABRION (C.), 1977. - Etude expérimentale du développement larvaire d'Hymenolepis stylosa (Rudolphi, 1809) Raillet, 1899 (Cestode, Cyclophyllide). Ann. Parasitol. hum. comp., 52, 117-130.

Gabrion (C.) et Gabrion (J.), 1976. - Etude ultrastructurale de la larve de Anomotaenia constricta (Cestoda, Cyclophyllidea). Z. Parasitenk., 49, 161-177.

HAY (E. D.) et Coward (S. J.), 1975. - Fine structure studies on the planarian, Dugesia. I. Nature of the \&Neoblast» and other cell types in Noninjured worms. J. Ultrastruct. Res., 50, 1-21.

JHA et SмYTH, 1969. - Ultrastructure of microtriches in Echinococcus granulosus. Exp. Parasitol., 25, 232-244.

Lascano (E. F.), Coltorti (E. A.) et Varela-Diaz (V. M.), 1975. - Fine structure of the germinal membrane of Echinococcus granulosus cysts. J. Parasitol., 61, 853-860.

Lumsden (R. D.), 1966. - Cytological studies on the absorptive surfaces of cestodes. I. The fine structure of the strobilar integument. Z. Parasitenk., 27, 355-382. 
Lumsden (R. D.) et Byram III (J.), 1967. - The ultrastructure of cestode muscle. J. Parasitol., 53, 326-342.

Moczon (T.), 1973. - Histochemical studies on the enzymes of Hymenolepis diminuta (Rud., 1819) (Cestoda). I. Some oxidoreductases in oncospheres and cysticercoids. Acta Parasitol. Pol., 21, 85-97.

Moczon (T.), 1973. - Histochemical studies on the enzymes of Hymenolepis diminuta (Rud., 1819) (Cestoda). II. Non-specific and specific phosphatases in oncospheres and cysticercoids. Acta Parasitol. Pol., 21, 99-107.

Mount (P. M.), 1970. - Histogenesis of the rostellar hooks of Taenia crassiceps (Zeder, 1800) (Cestoda). J. Parasitol., 56, 947-961.

Rees (G.), 1973. - The ultrastructure of the cysticercoid of Tatria octacantha Rees, 1973 (Cyclophyllidea: Amabiliidae) from the haemocoele of the damsel-fly nymphs Pyrrhosoma nymphula, Sulz and Enallagma cyathigerum, Charp. Parasitology, 67, 85-103.

SCHILler (E. L.), 1959. - Experimental studies on morphological variation in the cestode genus Hymenolepis. I. Morphology and development of the cysticercoid of $H$. nana in Tribolium confusum. Exp. Parasitol., 8, 91-118.

Sakamoto (T.) et Sugimura (M.), 1970. - Studies on echinococcosis XXIII. Electron microscopical observations on histogenesis of larval Echinococcus multilocularis. Jpn. J. Vet. Res., 18, 131-144.

Ubelaker (J. E.), Cooper (N. B.) et Allison (V. F.), 1970. - The fine structure of the cysticercoid of Hymenolepis diminuta. I. The outer wall of the capsule. Z. Parasitenk., 34, 258-270.

Voge (M.) et Heyneman (D.), 1957. - Development of Hymenolepis nana and Hymenolepis diminuta (Cestoda: Hymenolepididae) in the intermediate host Tribolium confusum. Univ. Calif. Publ. Zool., 59, 549-579. 